\title{
ON THE HAUSDORFF MEASURE OF THE JULIA SET AND THE ESCAPING SET OF ENTIRE FUNCTIONS WITH REGULARLY GROWING MAXIMUM MODULUS
}

\author{
Jie Ding \\ Taiyuan University of Technology, School of Mathematics, Taiyuan, Shanxi, 030024, P. R. China \\ and Christian-Albrechts-Universität zu Kiel, Mathematisches Seminar \\ Kiel, D-24098, Germany; dingjie@tyut.edu.cn
}

\begin{abstract}
We prove that the Hausdorff measure of the escaping set and the Julia set of an entire function $f$ is infinite with respect to certain gauge functions, provided that $f$ is outside of the Eremenko-Lyubich class, and that the maximum modulus $M(r, f)$ of $f$ satisfies a certain regularity condition.
\end{abstract}

\section{Introduction}

Let $f$ be a transcendental entire function, and denote by

$$
f^{n}:=\underbrace{f \circ \cdots \circ f}_{n}
$$

the $n$-th iterate of $f$, for $n \in \mathbf{N}$. The Fatou set $F(f)$ is the set of points in $\mathbf{C}$ such that $\left\{f^{n}\right\}$ forms a normal family in the sense of Montel (or, equivalently, is equicontinuous). The complement $J(f)$ of $F(f)$ is called the Julia set of $f$. Both sets are completely invariant. For an introduction to the basic properties of these sets, we refer to the survey [4] and the books [3, 18, 26].

A gauge function is a monotonically increasing function $h:[0, \varepsilon) \rightarrow[0,+\infty)$ which is continuous from the right and satisfies $h(0)=0$.

Definition 1.1. Let $A \subset \mathbf{R}^{n}$ be a set, $\delta>0$ a constant, and let $h$ be a gauge function. Then we call

$$
H^{h}(A):=\liminf _{\delta \rightarrow 0}\left\{\sum_{j=1}^{\infty} h\left(\operatorname{diam}\left(A_{j}\right)\right): A \subset \bigcup_{j=1}^{\infty} A_{j} \text { and } \operatorname{diam}\left(A_{j}\right)<\delta\right\}
$$

the Hausdorff measure with respect to $h$, where

$$
\operatorname{diam}\left(A_{j}\right)=\sup _{x, y \in A_{j}}|x-y|
$$

is the diameter of $A_{j}$.

The Hausdorff measure is an outer measure for measurable sets. In particular, when $h^{s}(r)=r^{s}(s>0)$, then $H^{h^{s}}(A)$ is the $s$-dimension Hausdorff measure of $A$. If $H^{h^{s}}(A)<\infty$ and $t>s$, then $H^{h^{t}}(A)=0$; if $H^{h^{s}}(A)>0$ and $t<s$, then $H^{h^{t}}(A)=\infty$. Moreover, there exists a constant $s$ such that $H^{h^{t}}(A)=0$ for all $t>s$

https://doi.org/10.5186/aasfm.2017.4215

2010 Mathematics Subject Classification: Primary 37F10, 30D05.

Key words: Entire function, escaping set, Julia set, Hausdorff measure, gauge function.

Financially supported by the NSFC (No. 11601362); Shanxi Scholarship Council of China (No. 2013-045); the Foundation Research Project of Shanxi Province (No. 2014021009-3) and the China Scholarship Council (No. 201306935005). 
and $H^{h^{t}}(A)=\infty$ for all $t<s$. The above $s$ is called Hausdorff dimension of $A$, and we denote $s=\operatorname{dim}(A)$.

In 1987, McMullen [17] proved that $\operatorname{dim}\left(J\left(E_{\lambda}\right)\right)=2$ for $\lambda \neq 0$, where $E_{\lambda}=$ $\lambda \exp (z)$. He also remarked that $H^{h}\left(J\left(E_{\lambda}\right)\right)=\infty$ when $h(t)=t^{2} \log ^{m}\left(\frac{1}{t}\right)$ and $m \in \mathbf{N}$. In his proofs, he first showed that these results hold for the escaping set $I(f):=$ $\left\{z ; f^{n}(z) \rightarrow \infty\right.$ as $\left.n \rightarrow \infty\right\}$, and then $I(f) \subset J(f)$ for the functions $E_{\lambda}$.

There is a close relation between the Julia set and the escaping set $I(f)$, which is studied for a general transcendental entire function $f$ by Eremenko. In [10], he proved that

$$
J(f)=\partial I(f) .
$$

Let $\operatorname{sing}\left(f^{-1}\right)$ denote the set of singular values of $f$, which consists of critical and finite asymptotic values. The Eremenko-Lyubich class

$$
\mathcal{B}:=\left\{f \text { is transcendental entire function: } \operatorname{sing}\left(f^{-1}\right) \text { is bounded }\right\}
$$

plays an important role in complex dynamics. In [11], Eremenko and Lyubich introduced a logarithmic change of variable, which has become a standard tool for studying entire functions in class $\mathcal{B}$. Using this method, they showed that $I(f) \subset J(f)$ for $f \in \mathcal{B}$. It is easy to check that $E_{\lambda} \in \mathcal{B}$, thus $I\left(E_{\lambda}\right) \subset J\left(E_{\lambda}\right)$.

There are many results on the Hausdorff dimension of entire functions, see $[2$, $5,6,21,22,24,25]$. In [2] and [22], Barański and Schubert independently proved that $\operatorname{dim}(J(f))=2$ if $f \in \mathcal{B}$ has finite order of growth. For more details, we refer to surveys $[14,23]$.

Given $\lambda_{0} \in(0,1 / e)$, the function $E_{\lambda_{0}}$ has two fixed points $\alpha_{\lambda_{0}}$ and $\beta_{\lambda_{0}}$, where $\alpha_{\lambda_{0}}$ is attracting and $\beta_{\lambda_{0}}>e$ is repelling. Recall that a classical result of Koenigs says that there exists a function $\Phi_{\lambda_{0}}$ holomorphic in a neighborhood $D\left(\lambda_{0}\right)$ of $\beta_{\lambda_{0}}$ which satisfies $\Phi_{\lambda_{0}}\left(\beta_{\lambda_{0}}\right)=0, \Phi_{\lambda_{0}}^{\prime}\left(\beta_{\lambda_{0}}\right)=1$ and

$$
\Phi_{\lambda_{0}}\left(E_{\lambda_{0}}(z)\right)=\beta_{\lambda_{0}} \Phi_{\lambda_{0}}(z), \quad z, E_{\lambda_{0}}(z) \in U
$$

It is easy to see that $\Phi_{\lambda_{0}}(x) \in \mathbf{R}$ for $x \in \mathbf{R}$. Recently, Peter [19, 20] studied the Hausdorff measure on Julia set of exponential functions and entire functions in class $\mathcal{B}$ by introducing such a $\Phi$ and proving the next result.

Theorem A. Define $\lambda_{0} \in(0,1 / e), \beta_{\lambda_{0}}, \Phi_{\lambda_{0}}$ as above, let $K_{\lambda_{0}}=\log 2 / \log \beta_{\lambda_{0}}$ and $h(t)=t^{2} g(t)$ be a gauge function. If

$$
\liminf _{t \rightarrow 0} \frac{\log g(t)}{\log \Phi_{\lambda_{0}(1 / t)}}>K_{\lambda_{0}}
$$

then $H^{h}\left(J\left(E_{\lambda}\right)\right)=\infty$ for all $\lambda \in \mathbf{C} \backslash\{0\}$.

Theorem B. Let $\lambda_{0} \in(0,1 / e)$. There exists $K>0$ with the following property: If $f \in \mathcal{B}$ and $\rho(f)=\rho>1 / 2$, then $H^{h}(J(f))=\infty$, where $h(t)=t^{2}\left(\Phi_{\lambda_{0}}(1 / t)\right)^{\kappa}$ and $\kappa>(\log (\rho)+K) / \log \beta_{\lambda_{0}}$.

Remark 1.2. Peter [20] has obtained a necessary condition for a gauge function $h^{\prime}$ such that $H^{h^{\prime}}\left(J\left(e^{\lambda z}\right)\right)=0$.

Bergweiler and Karpińska [5] considered entire functions $f \notin \mathcal{B}$ for which there exist constant $A, B, C, r_{0}>1$ such that

$$
A \log M(r, f) \leq \log M(C r, f) \leq B \log M(r, f) \text { for all } r>r_{0},
$$

and proved the following result. 
Theorem C. If $f$ is an entire function satisfying (1.2), then $\operatorname{dim}(I(f) \cap J(f))=$ 2 .

\section{Main results}

The first result is in the spirit of Theorem A, but for functions $f$ satisfying (1.2).

Theorem 2.1. Let $\lambda_{0} \in(0,1 / e)$ and $\beta_{\lambda_{0}}, \Phi_{\lambda_{0}}$ be as above. Let $\Delta>0$ be a constant and $\kappa>\frac{\log (1 / \Delta)}{\log \beta_{\lambda_{0}}}$. If $h(t)=t^{2} g(t)$ is a gauge function satisfying

$$
\liminf _{t \rightarrow 0} \frac{\log g(t)}{\log \Phi_{\lambda_{0}}(1 / t)}>\kappa
$$

then for every entire function $f$ satisfying (1.2), we have $H^{h}(I(f) \cap J(f))=\infty$.

Corollary 2.2. Let $f$ be an entire function satisfying (1.2), and let $h(t)=$ $t^{2} \log ^{m} \frac{1}{t}$ for $m \in \mathbf{N}$. Then $H^{h}(I(f) \cap J(f))=\infty$.

As is mentioned in [5], the hypothesis (1.2) is satisfied if there exist constants $c_{1}, c_{2}, \rho>0$ such that

$$
c_{1} r^{\rho} \leq \log M(r, f) \leq c_{2} r^{\rho},
$$

for large $r$, and thus in particular if there exist $c, \rho>0$ such that

$$
\log M(r, f) \sim c r^{\rho},
$$

as $r \rightarrow \infty$. Hence there are many entire functions satisfying the condition (1.2). We may prove this assertion by relying on the following theorem of Clunie [9]: Let $\phi(r)$ be increasing and convex in $\log r$ with $\phi(r) \neq O(\log r)(r \rightarrow \infty)$. (This condition is imposed to exclude certain trivial cases.) Then there is an entire function $f(z)$ such that

$$
\log M(r, f) \sim \phi(r) \text { and } T(r, f) \sim \phi(r)
$$

as $r \rightarrow \infty$.

Theorem 2.3 below is of crucial importance. We define the set $T(f, \alpha, \beta, \delta, \lambda)$ consisting of points $z$ such that

$$
\begin{aligned}
\alpha \log M(|z|, f) & \leq\left|\frac{z f^{\prime}(z)}{f(z)}\right| \leq \beta \log M(|z|, f), \\
|f(z)| & \geq \exp \left(|z|^{\delta}\right),
\end{aligned}
$$

and

$$
\left|\frac{\zeta f^{\prime}(\zeta)}{f(\zeta)}\right| \leq \beta \log M(|\zeta|, f), \quad \text { for }|\zeta-z| \leq \lambda \frac{|z|}{\log M(|z|, f)} .
$$

In this definition, the conditions (2.1) and (2.3) are the same as those appearing in [5]. Condition (2.2) concerning the escaping rate of point $z$ is different from the one in [5]. Indeed, we consider a subset with much faster escaping rate.

For $R>0$, let $A(R)=\{z \in \mathbf{C}: R<|z|<2 R\}$. For measurable sets $X, Y \subset \mathbf{C}$ the density of $X$ in $Y$ is defined by

$$
\operatorname{dens}(X, Y)=\frac{\operatorname{area}(X \cap Y)}{\operatorname{area}(Y)} .
$$

Theorem 2.3. Let $f$ be an entire function satisfying (1.2). Then there exist positive constants $\alpha, \beta, \delta$ and $\eta$ such that if $\lambda \geq 0$, we have dens $(T(f, \alpha, \beta, \delta, \lambda), A(R))>$ $\eta$ for all sufficiently large $R$. 


\section{Proof of Theorem 2.3}

Throughout this article, denote by $T(r, f), M(r, f)$ and $L(r, f)$ the Nevanlinna characteristic function, maximum modulus and minimum modulus of $f$, respectively. By $n(r, a)$ we denote the number of zeros of $f-a$ in the disc $\{z:|z|<r\}$. For an entire function $f$, the growth order $\rho(f)$ and lower order $\lambda(f)$ are respectively defined as

$$
\rho(f)=\limsup _{r \rightarrow \infty} \frac{\log T(r, f)}{\log r} \text { and } \quad \lambda(f)=\liminf _{r \rightarrow \infty} \frac{\log T(r, f)}{\log r} .
$$

For more details, we refer the reader to the books $[12,13]$.

\subsection{Some lemmas.}

Lemma 3.1. [5, Theorem 1.2] Let $f$ be an entire function satisfying (1.2). Then there exist $\alpha_{0}, \beta_{0}$ and $\eta_{0}$ such that dens $\left(T\left(f, \alpha_{0}, \beta_{0}, 0, \lambda\right), A(R)\right)>\eta_{0}$ for all large enough $R$.

Lemma 3.2. [12, Theorem 3.4] (Borel Theorem) The order of the Weierstrass canonical product $f(z)$ is equal to the order of $n(r, 0)$, i.e.,

$$
\rho(f)=\limsup _{r \rightarrow \infty} \frac{\log n(r, 0)}{\log r} .
$$

Lemma 3.3. [12, Theorem 3.2] Let $\left\{\alpha_{k}\right\}, k=1,2, \ldots$, be a sequence of complex numbers satisfying $0<\left|\alpha_{1}\right| \leq\left|\alpha_{2}\right| \leq \ldots$, such that

$$
\sum_{k=1}^{\infty} \frac{1}{\left|\alpha_{k}\right|^{p}}<\infty,
$$

where $p$ is a positive integer. Suppose that an entire function $g(z)$ has a power series representation of the form

$$
g(z)=1+c_{p} z^{p}+c_{p+1} z^{p+1}+\cdots
$$

Then the product

$$
f(z)=\prod_{k=1}^{\infty} g\left(\frac{z}{\alpha_{k}}\right)
$$

converges absolutely and uniformly on each bounded disc to an entire function.

Lemma 3.4. [16, Theorem 1] Given $n$ points $\alpha_{1}, \alpha_{2}, \ldots, \alpha_{n}$ of the complex plane (repetitions being allowed), and an arbitrary number $H>0$, there exists a set of at most $n$ circles, whose radii $h_{k}$ satisfy the inequality

$$
\sum_{k=1}^{n} h_{k}^{2} \leq 4 H^{2}
$$

with the property that, if $z$ is outside these circles, then

$$
\sum_{k=1}^{n} \frac{1}{\left|z-\alpha_{k}\right|} \leq 2 \frac{n}{H} .
$$

Lemma 3.5. [15, p. 19] (Boutrox-Cartan lemma) Given any constant $H>0$ and complex numbers $\alpha_{1}, \alpha_{2}, \ldots, \alpha_{n}$, there is a series of circles in the complex plane, with the sum of the radii equal to $2 H$, such that for each point $z$ lying outside these circles,

$$
\left|z-\alpha_{1}\right|\left|z-\alpha_{2}\right| \cdots\left|z-\alpha_{n}\right|>(H / e)^{n}
$$


For an entire function $f$ which satisfies the regularly growth condition (1.2), the order is bounded from above and the lower order is bounded from below.

Lemma 3.6. [5, p. 533] Let $f$ be an entire function satisfying (1.2). Then

$$
0<\frac{\log A}{\log C} \leq \lambda(f) \leq \rho(f) \leq \frac{\log B}{\log C}<\infty,
$$

and there exists a constant $K>0$ such that

$$
n(2 r, a) \leq K n(r, a) .
$$

3.2. The proof. Lemma 3.1 implies that the theorem holds in the special case $\delta=\lambda=0$.

Replacing $f$ with $f-a$, if necessary, we may assume that 0 is not a Valiron deficiency of $f$. By the discussions in [5], we can find a constant $C>16$ such that $n(C r, 0)>2 n(r, 0)$. Given $\theta \in(0,1)$, we may thus choose a subsequence of $\left\{z_{j}\right\}$ of zeros of $f$ such that there are $\left[\theta n\left(C^{k} r_{0}, 0\right)\right]$ zeros in the annuli $A_{k}=\left\{z: C^{k+1} r_{0}>\right.$ $\left.|z| \geq C^{k} r_{0}\right\}$, denoted by $\left\{z_{j(k)}\right\}$, for $r_{0}>1$ and all $k \in \mathbf{N}$. Then

$$
\begin{aligned}
\sum_{j} \frac{1}{\left|z_{j}\right|} & =\sum_{k=1}^{\infty} \sum_{z_{j(k)} \in A_{k}} \frac{1}{\left|z_{j(k)}\right|} \leq \sum_{k=1}^{\infty} \frac{\theta n\left(C^{k} r_{0}, 0\right)}{C^{k} r_{0}}=\sum_{k=1}^{\infty} \frac{\theta(1+\theta) n\left(C^{k-1} r_{0}, 0\right)}{C^{k} r_{0}} \\
& =\sum_{k=1}^{\infty} \frac{\theta(1+\theta)^{2} n\left(C^{k-2} r_{0}, 0\right)}{C^{k} r_{0}}=\sum_{k=1}^{\infty} \frac{\theta(1+\theta)^{k} n\left(r_{0}, 0\right)}{C^{k} r_{0}} \\
& =\frac{\theta n\left(r_{0}, 0\right)}{r_{0}} \sum_{k=1}^{\infty} \frac{(1+\theta)^{k}}{C^{k}} \leq \frac{\theta n\left(r_{0}, 0\right)}{r_{0}} \sum_{k=1}^{\infty}\left(\frac{1}{8}\right)^{k}<\infty .
\end{aligned}
$$

Consequently, $h(z)=\prod_{k=1}^{\infty}\left(1-\frac{z}{z_{j}}\right)$ is an entire function by Lemma 3.3. Recall that $r>r_{0}$. Hence there exists an integer $k$ such that $C^{k} r_{0} \leq r<C^{k+1} r_{0}$. It follows from Lemma 3.2 that

$$
\begin{aligned}
\rho(h) & =\limsup _{r \rightarrow \infty} \frac{\log n(r, 0)}{\log r} \leq \limsup _{k \rightarrow \infty} \frac{\log n\left(C^{k+1} r_{0}, 0\right)}{\log \left(C^{k} r_{0}\right)} \\
& =\limsup _{k \rightarrow \infty} \frac{\log (1+\theta)^{k+1} n\left(r_{0}, 0\right)}{\log \left(C^{k} r_{0}\right)}=\frac{\log (1+\theta)}{\log C},
\end{aligned}
$$

while

$$
\begin{aligned}
\rho(h) & =\limsup _{r \rightarrow \infty} \frac{\log n(r, 0)}{\log r} \geq \limsup _{k \rightarrow \infty} \frac{\log n\left(C^{k} r_{0}, 0\right)}{\log \left(C^{k+1} r_{0}\right)} \\
& =\limsup _{k \rightarrow \infty} \frac{\log (1+\theta)^{k} n\left(r_{0}, 0\right)}{\log \left(C^{k+1} r_{0}\right)}=\frac{\log (1+\theta)}{\log C} .
\end{aligned}
$$

Then

$$
\rho(h)=\frac{\log (1+\theta)}{\log C}<\frac{\log 2}{\log 16}<1 .
$$

If $\theta$ is sufficiently small, then Lemma 3.6 implies $\rho(h)<\frac{\log A}{\log C} \leq \lambda(f)$. Hence, the definitions of $\rho(h)$ and $\lambda(h)$ yield

$$
T(r, h)=o(T(r, f)),
$$

as $r \rightarrow \infty$ without an exceptional set. 
Let

$$
g(z)=\frac{f(z)}{h(z)} .
$$

Using the formula (6.6) in [12, p. 29] and the Nevanlinna first fundamental theorem, we have $T(r, g) \leq T(r, f)+T(r, h)=O(T(r, f))$. Moreover, from the standard inequalities $T(r, f) \leq \log ^{+} M(r, f) \leq 3 T(2 r, f)$, we can deduce that

$$
\log M(r, g)=O(\log M(2 r, f)), \quad \text { as } r \rightarrow \infty .
$$

So $g$ satisfies the assumption of Lemma 3.1 with the constants $A$ and $B$ being chosen suitably. That is,

$$
\operatorname{dens}\left(T\left(g, \alpha^{*}, \beta^{*}, 0,0\right), A(R)\right) \geq \eta
$$

for sufficiently large $R$ and for $0<\alpha^{*}<\alpha_{0}, \beta^{*}>\beta_{0}$.

Now $\frac{z g^{\prime}(z)}{g(z)}=\frac{z f^{\prime}(z)}{f(z)}-\frac{z h^{\prime}(z)}{h(z)}$, so that

$$
\left|\frac{z g^{\prime}(z)}{g(z)}\right|-\left|\frac{z h^{\prime}(z)}{h(z)}\right| \leq\left|\frac{z f^{\prime}(z)}{f(z)}\right| \leq\left|\frac{z g^{\prime}(z)}{g(z)}\right|+\left|\frac{z h^{\prime}(z)}{h(z)}\right| .
$$

Since $h(0)=1$, it follows from the formula (1.3') in [12, p. 88] that

$$
\left|\frac{h^{\prime}(z)}{h(z)}\right| \leq \frac{4 s T(s, h)}{(s-|z|)^{2}}+\sum_{\left|z_{j}\right|<s} \frac{2}{\left|z-z_{j}\right|}
$$

for $s>|z|$. Considering $s=2|z|$, we get, for every $z \in A(R)$ and $\varepsilon>0$,

$$
\frac{4 s T(s, h)}{(s-|z|)^{2}} \leq \frac{8 T(2|z|, h)}{|z|}=O\left(r^{\rho(h)-1+\varepsilon}\right),
$$

where $r=|z|$. Applying Lemma 3.4 to $\sum_{\left|z_{j}\right|<s} \frac{2}{\left|z-z_{j}\right|}$ with $H=\frac{\sqrt{3 \epsilon} R}{2}$, where $\epsilon$ is a small constant, we deduce that

$$
\sum_{\left|z_{j}\right|<s} \frac{2}{\left|z-z_{j}\right|} \leq \frac{2 n(2 r, 0)}{H}=O\left(\frac{T(2 r, h)}{r}\right)=O\left(r^{\rho(h)-1+\varepsilon}\right),
$$

for all $z$ outside set $E_{1}$ which is a union of finite discs and satisfies $\operatorname{area}\left(E_{1}\right)=$ $\epsilon$ area $A(R)$. So,

$$
\left|\frac{z h^{\prime}(z)}{h(z)}\right|=O\left(|z|^{\rho(h)+\varepsilon}\right)=o(T(r, f))=o(\log M(r, f)),
$$

for all $z \in T\left(g, \alpha^{*}, \beta^{*}, 0,0\right) \backslash E_{1}$. Thus, there are constants $\alpha^{* *}, \beta^{* *}$ such that

$$
\alpha^{* *} \log M(|z|, f) \leq\left|\frac{z f^{\prime}(z)}{f(z)}\right| \leq \beta^{* *} \log M(|z|, f)
$$

for all $z \in T\left(g, \alpha^{*}, \beta^{*}, 0,0\right) \backslash E_{1}$. Therefore,

$$
T\left(g, \alpha^{*}, \beta^{*}, 0,0\right) \backslash E_{1} \subset T\left(f, \alpha^{* *}, \beta^{* *}, 0,0\right) .
$$

For any $R$ and $z \in A(R)$, we have

$$
\begin{aligned}
h(z) & =\prod_{\left|z_{j}\right| \leq \frac{R}{4}}\left(1-\frac{z}{z_{j}}\right) \cdot \prod_{\frac{R}{4}<\left|z_{j}\right| \leq 4 R}\left(1-\frac{z}{z_{j}}\right) \cdot \prod_{\left|z_{j}\right|>4 R}\left(1-\frac{z}{z_{j}}\right) \\
& =h_{1}(z) h_{2}(z) h_{3}(z) .
\end{aligned}
$$


We now estimate $\log \left|h_{i}(z)\right|, i=1,2,3$, starting with

$$
\log \left|h_{1}(z)\right| \geq \sum_{\left|z_{j}\right| \leq \frac{R}{4}} \log \left(\left|\frac{z}{z_{j}}\right|-1\right) \geq \log (3) n\left(\frac{R}{4}, 0\right) .
$$

Using the Boutrox-Cartan lemma to $h_{2}(z)$, we get

$$
\left|h_{2}(z)\right|=\prod_{\frac{R}{4}<\left|z_{j}\right| \leq 4 R}\left|1-\frac{z}{z_{j}}\right| \geq \frac{1}{(4 R)^{m}}\left(\frac{H}{e}\right)^{m}=\left(\frac{H}{4 e R}\right)^{m}
$$

except for a set $E_{2}$ which is a union of disks with sum of radii less than $2 H$, where $m=n(4 R, 0)-n\left(\frac{R}{4}, 0\right)$. For a constant $c<8 e$, put $2 H=c R$. Thus

$$
\left|h_{2}(z)\right| \geq\left(\frac{H}{4 e R}\right)^{m} \geq\left(\frac{c}{8 e}\right)^{m} .
$$

For the above $R$ there must exist an integer $k$ satisfying $r_{k} \leq \frac{R}{4}<r_{k+1}$, where $r_{k}=C^{k} r_{0}$. Recall that $C>16$. Then $r_{k+2}=C r_{k+1}>4 R$ and

$$
\begin{aligned}
m & =n(4 R, 0)-n\left(\frac{R}{4}, 0\right) \leq n\left(r_{k+2}, 0\right)-n\left(r_{k}, 0\right) \\
& =\left(n\left(r_{k+2}, 0\right)-n\left(r_{k+1}, 0\right)\right)+\left(n\left(r_{k+1}, 0\right)-n\left(r_{k}, 0\right)\right) \\
& \leq \theta n\left(r_{k+1}, 0\right)+\theta n\left(r_{k}, 0\right) \leq \theta(\theta+2) n\left(r_{k}, 0\right) \leq \theta(\theta+2) n\left(\frac{R}{4}, 0\right) .
\end{aligned}
$$

From the above argument, we can get

$$
\log \left|h_{2}(z)\right| \geq-\log \left(\frac{8 e}{c}\right) m \geq-\varepsilon^{\prime} n\left(\frac{R}{4}, 0\right),
$$

where $\varepsilon^{\prime}=\log \left(\frac{8 e}{c}\right) \theta(2+\theta)>0$. Using the inequality $\log (1-x) \geq-x, x \leq \frac{1}{2}$, we obtain

$$
\log \left|h_{3}(z)\right| \geq \sum_{\left|z_{j}\right| \geq 4 R}\left(1-\frac{2 R}{\left|z_{j}\right|}\right) \geq-2 \sum_{\left|z_{j}\right| \geq 4 R} \frac{R}{\left|z_{j}\right|} .
$$

Noting that there exists a constant $k_{0}$ such that $r_{k_{0}} \leq 4 R<r_{k_{0}+1}$, we deduce

$$
\begin{aligned}
\sum_{\left|z_{j}\right| \geq 4 R} \frac{R}{\left|z_{j}\right|} & \leq \sum_{k=k_{0}}^{\infty} \sum_{z_{j} \in A_{k}} \frac{R}{\left|z_{j}\right|} \leq \sum_{k=k_{0}}^{\infty}\left(n\left(r_{k+1}, 0\right)-n\left(r_{k}, 0\right)\right) \frac{R}{r_{k}} \\
& \leq \sum_{k=k_{0}}^{\infty} \frac{R \theta(1+\theta)^{k-k_{0}}}{C^{k-k_{0}} r_{k_{0}}} n\left(r_{k_{0}}, 0\right) \\
& <\frac{C}{4} \sum_{k=k_{0}}^{\infty}\left(\frac{1+\theta}{C}\right)^{k-k_{0}} \theta n(4 R, 0) \leq \frac{2 C K^{4} \theta}{7} n\left(\frac{R}{4}, 0\right),
\end{aligned}
$$

where $K$ is the constant as in Lemma 3.6. Therefore,

$$
\log \left|h_{3}(z)\right|>-\varepsilon^{\prime \prime} n\left(\frac{R}{4}, 0\right),
$$

where $\varepsilon^{\prime \prime}=\frac{4 C K^{4}}{7} \theta$.

If $\theta$ is sufficiently small, then

$$
\log 3-\left(\log \left(\frac{8 e}{c}\right)(2+\theta)+\frac{4 C K^{4}}{7}\right) \theta=\gamma \geq \frac{1}{2} .
$$


It follows from (3.5), (3.6), (3.7) and (3.9) that $\log |h(z)| \geq \gamma n\left(\frac{R}{4}, 0\right)$ for all $z \in A(R)$ outside $E_{2}$. Furthermore, (3.1) implies that

$$
\log |h(z)| \geq \gamma|z|^{\delta} \geq \frac{1}{2}|z|^{\delta}
$$

where $\delta<\frac{\log (1+\theta)}{\log C}=\rho(h)$.

From above argument, for any $z \in T\left(g, \alpha^{*}, \beta^{*}, 0,0\right) \backslash E_{2}$, we have

$$
|f(z)|=|g(z) h(z)| \geq \exp \left(\frac{1}{2}|z|^{\delta}\right) .
$$

So $T\left(g, \alpha^{*}, \beta^{*}, 0,0\right) \backslash E \subset T\left(f, \alpha^{*}, \beta^{*}, \delta, 0\right)$, where $E=E_{1} \cup E_{2}$. This is the special case of Theorem 2.3. For the general $\lambda$, we can use the same argument as Theorem 1.2 in [5]. Thus we finish the proof of Theorem 2.3.

Remark 3.7. There are many lower estimates known for the modulus of entire functions of order $<1$, and, in particular, for functions of order $<1 / 2$ in the book [8]. For our use, a more precise estimate is required for the function $h$.

\section{Proof of main theorems}

4.1. Preparation. First, we recall the Koebe distortion theorem.

Lemma 4.1. (Koebe Distortion Theorem) Let $z_{0} \in \mathbf{C}, r>0$, and let $f$ be a univalent function in $D\left(z_{0}, r\right)$. If $z \in D\left(z_{0}, r\right)$, then

$$
r^{2}\left|f^{\prime}\left(z_{0}\right)\right| \frac{r-\left|z-z_{0}\right|}{\left(r+\left|z-z_{0}\right|\right)^{3}} \leq\left|f^{\prime}(z)\right| \leq r^{2}\left|f^{\prime}\left(z_{0}\right)\right| \frac{r+\left|z-z_{0}\right|}{\left(r-\left|z-z_{0}\right|\right)^{3}}
$$

and

$$
r^{2}\left|f^{\prime}\left(z_{0}\right)\right| \frac{\left|z-z_{0}\right|}{\left(r+\left|z-z_{0}\right|\right)^{2}} \leq\left|f(z)-f\left(z_{0}\right)\right| \leq r^{2}\left|f^{\prime}\left(z_{0}\right)\right| \frac{\left|z-z_{0}\right|}{\left(r-\left|z-z_{0}\right|\right)^{2}} .
$$

For our use, we also need the following consequence of Lemma 4.1.

Lemma 4.2. Let $\Omega$ be a domain, and let $Q \subset \Omega$ be compact. Then there exists a constant $C^{\prime}>0$ such that if $f$ is univalent in $\Omega$ and $z, \xi \in Q$, then $\left|f^{\prime}(\xi)\right| \leq C^{\prime}\left|f^{\prime}(z)\right|$.

Lemma 4.3 below plays an important role in proving that Hausdorff measure is $\infty$. Before stating it, consider, for $l \in \mathbf{N}$, a collection $\mathcal{A}_{l}$ of compact, disjoint and connected subsets of $\mathbf{C}$ with positive Lebesgue measure. Let $A_{l}$ be the union of all elements of $\mathcal{A}_{l}$. We say that $\left\{\mathcal{A}_{l}\right\}$ is a series nesting intersection sets if it satisfies the following properties:

(a) Every element of $\mathcal{A}_{l+1}$ is contained in a unique element of $\mathcal{A}_{l}$.

(b) Every element of $\mathcal{A}_{l}$ contains at least one element of $\mathcal{A}_{l+1}$.

(c) For any $F \in \mathcal{A}_{l}$, there exist two sequences of positive numbers $\left\{\Delta_{l}\right\}$ and $\left\{d_{l}\right\}\left(d_{l} \rightarrow 0\right)$ such that

$$
\operatorname{dens}\left(A_{l+1}, F\right) \geq \Delta_{l} ; \quad \operatorname{diam} F \leq d_{l} .
$$

The intersection $A=\bigcap_{l=1}^{\infty} A_{l}$ is a non-empty and compact set.

Lemma 4.3. [20, Lemma 3.3] Let $\left\{\mathcal{A}_{l}\right\}, A,\left\{d_{l}\right\},\left\{\Delta_{l}\right\}$ be as above. Let $\varepsilon>0$ and $\varphi:(0, \varepsilon) \rightarrow \mathbf{R}_{\geq 0}$ be a decreasing continuous function such that $t^{2} \varphi(t)$ is increasing. 
Further, suppose that $\lim _{t \rightarrow 0} t^{2} \varphi(t)=0$ and

$$
\lim _{l \rightarrow \infty} \varphi\left(d_{l}\right) \prod_{j=1}^{l} \Delta_{j}=\infty .
$$

Define $h:[0, \varepsilon) \longrightarrow \mathbf{R}$ by setting

$$
h(t)= \begin{cases}t^{2} \varphi(t), & t>0, \\ 0, & t=0 .\end{cases}
$$

Then $h(t)$ is a continuous gauge function, and $H^{h}(A)=\infty$.

Let $L$ be a constant such that $\log M(2 r, f) \leq L \log M(r, f)$ and let $t(R)=$ $\frac{\lambda R}{L \log M(R, f)}$. Bergweiler and Karpińska [5] applied the Ahlfors three islands theorem to domains

$$
D_{v}=\{z \in \mathbf{C}:|\Re z|<1,|\Im z-8 \pi v|<3 \pi\}, \quad v=1,2,3,
$$

and showed that

Lemma 4.4. [5, Lemma 5.1] Let $a \in T(f, \alpha, \beta, \delta, \lambda) \cap A(R)$ and $v \in\{1,2,3\}$. If $R$ is sufficiently large, then $D(a, t(R))$ contains a subdomain $U$ such that $\log f$ maps $U$ bijectively onto one of the domains

$$
\begin{aligned}
\Omega_{v}(a) & =\log f(a)+D_{v} \\
& =\{z \in \mathbf{C}:|\Re z-\log f(a)|<1,|\Im z-\log f(a)-8 \pi v|<3 \pi\} .
\end{aligned}
$$

Moreover, there exist $\tau, q$ such that if $V$ is the subset of $U$ which is mapped onto

$$
Q_{v}(a)=\{z \in \mathbf{C}: 0 \leq(\Re z-\log f(a))<\log 2,|\Im z-\log f(a)-8 \pi v| \leq 2 \pi\},
$$

then

$$
\operatorname{area}(V) \geq \tau t(R)^{2}
$$

and

$$
\left|\frac{f^{\prime}(z)}{f(z)}\right| \geq \frac{q}{t(R)} \quad \text { for } z \in V .
$$

The following lemma concerns with the number of discs $D(a, t(R))$ in the annulus $A(R)=\{z \in \mathbf{C}: R<|z|<2 R\}$.

Lemma 4.5. [5, Lemma 5.2] Let $\eta$ be as in Theorem 2.3. For sufficiently large $R$ there exists $m(R) \in \mathbf{N}$ satisfying

$$
m(R) \geq \frac{\eta R^{2}}{2 t^{2}(R)}
$$

such that there are $m(R)$ points $a_{j} \in T(f, \alpha, \beta, \delta, \lambda) \cap A(R), j=1,2, \cdots, m(R)$, satisfying $D\left(a_{j}, t(R)\right) \subset A(R)$ for all $j$ and $D\left(a_{j}, t(R)\right)$ are pairwise disjoint.

Lemma 4.6. [27, Corollary 5] Let $f$ be a transcendental meromorphic function with at most finitely many poles, and let $d>1$ be a constant. If for all sufficiently large $R>0$, we have

$$
\log M(2 R, f)>d \log M(R, f),
$$

then $J(f)$ has an unbounded component, and all components of $F(f)$ are simply connected. 
4.2. Proof of Theorem 2.1. The idea of construction of sets $\mathcal{A}_{l}$ and part of the proof is from that of Theorem 2.1 in [5]. For completeness, we repeat it here.

Choose $R_{0}$ large enough, and let

$$
\mathcal{A}_{0}=\left\{A\left(R_{0}\right)\right\}
$$

By Lemma 4.4, there are domains $D\left(a\left(R_{0}\right), t\left(R_{0}\right)\right)\left(\subset A\left(R_{0}\right)\right), U\left(a\left(R_{0}\right)\right)$ and $V\left(a\left(R_{0}\right)\right)$ with $V\left(a\left(R_{0}\right)\right) \subset U\left(a\left(R_{0}\right)\right) \subset D\left(a\left(R_{0}\right), t\left(R_{0}\right)\right)$ such that $\log f$ maps $U\left(a\left(R_{0}\right)\right)$ onto the rectangles $\Omega_{v}\left(a\left(R_{0}\right)\right)$ and $V\left(a\left(R_{0}\right)\right)$ onto $Q_{v}\left(a\left(R_{0}\right)\right)$. Since $f=\exp (\log f)$, we obtain that $f\left(U\left(a\left(R_{0}\right)\right)\right)$ and $f\left(V\left(a\left(R_{0}\right)\right)\right)$ are the annuli $\left\{z:\left|f\left(a\left(R_{0}\right)\right)\right| / e<|z|<\right.$ $\left.e\left|f\left(a\left(R_{0}\right)\right)\right|\right\}$ and $A\left(\left|f\left(a\left(R_{0}\right)\right)\right|\right)$, respectively.

By Lemma 4.5 , we note that there are at least $m\left(R_{0}\right) \geq \frac{\eta R_{0}^{2}}{2 t^{2}\left(R_{0}\right)}$ many disjoint discs, say $\left\{D\left(a_{j_{1}}\left(R_{0}\right), t\left(R_{0}\right)\right)\right\}_{j=1}^{m\left(R_{0}\right)}$, that are contained in $A\left(R_{0}\right)$. Consequently, there are $m\left(R_{0}\right)$ disjoint $V\left(a_{j_{1}}\left(R_{0}\right)\right)$ having the above properties. Now, we can construct the sets

$$
\mathcal{A}_{1}=\left\{V\left(a_{j_{1}}\left(R_{0}\right)\right): 1 \leq j \leq m\left(R_{0}\right)\right\} .
$$

For some $j$, put $R_{1, V_{j_{1}}}=R_{1, V\left(a_{j_{1}}\left(R_{0}\right)\right)}=\left|f\left(a_{j_{1}}\left(R_{0}\right)\right)\right|$. Since $R_{0}$ is large enough, it follows from (2.2) that $R_{1, V_{j_{1}}}>R_{0}$. Let $D\left(a\left(R_{1, V_{j_{1}}}\right), t\left(R_{1, V_{j_{1}}}\right)\right)$ be a disc contained in $A\left(R_{1, V_{j_{1}}}\right)$. Using Lemma 4.4 again, there are domains $V\left(a\left(R_{1, V_{j_{1}}}\right)\right)$ and $U\left(a\left(R_{1, V_{j_{1}}}\right)\right)$ such that $V\left(R_{1, V_{j_{1}}}\right) \in U\left(a\left(R_{1, V_{j_{1}}}\right)\right) \in D\left(a\left(R_{1, V_{j_{1}}}\right), t\left(R_{1, V_{j_{1}}}\right)\right)$. Therefore, $V\left(a\left(R_{1, V_{j_{1}}}\right)\right)$ and $U\left(a\left(R_{1, V_{j_{1}}}\right)\right)$ are mapped by $\log f$ bijectively onto $Q_{v}\left(a\left(R_{1, V_{j_{1}}}\right)\right)$ and $\Omega_{v}\left(a\left(R_{1, V_{j_{1}}}\right)\right)$ respectively. Then $\log f^{2}$ is a bijective mapping from a subset of $V_{j_{1}}\left(R_{0}\right)$ onto $Q_{v}\left(a\left(R_{1, V_{j_{1}}}\right)\right)$.

We define

$$
\mathcal{A}_{2}=\bigcup_{V_{j_{1}} \in \mathcal{A}_{1}}\left\{\psi_{V_{j_{1}}}\left(Q_{v}\left(a_{j_{2}}\left(R_{1, V_{j_{1}}}\right)\right)\right): 1 \leq j_{2} \leq m\left(R_{1, V_{j_{1}}}\right)\right\}
$$

where $\psi_{V_{j_{1}}}$ is the inverse function of $\log f^{2}$ restricted on $V_{j_{1}}$ and $m\left(R_{1, V_{j_{1}}}\right)$ is the number of domain $V\left(a\left(R_{1, V_{j_{1}}}\right)\right)$ in $A\left(R_{1, V_{j_{1}}}\right)$.

Inductively, $\mathcal{A}_{l}$ consists of all sets $F$ which satisfy $f^{l}(F)=A\left(R_{l, F}\right)$, where $R_{l, F}>R_{0}$. If $G$ is an element of $\mathcal{A}_{l-1}$ which contains $F$, then for some $j \in$ $\left\{1,2, \cdots, m\left(R_{l-1, G}\right)\right\}$, we have

$$
f^{l-1}(F)=V_{j}\left(R_{l-1, G}\right) \subset D\left(a_{j}\left(R_{l-1, G}\right), t\left(R_{l-1, G}\right) \subset A\left(R_{l-1, G}\right)=f^{l-1}(G) .\right.
$$

Now we will construct $\mathcal{A}_{l+1}$. By Lemma 4.4, there exists a domain

$$
U\left(a_{j}\left(R_{l-1, G}\right)\right) \subset D\left(a_{j}\left(R_{l-1, G}\right), t\left(R_{l-1, G}\right)\right),
$$

which is mapped by $\log f$ bijectively onto $\Omega_{v}\left(a_{j}\left(R_{l-1, G}\right)\right)$ and its subset $V_{j}\left(R_{l-1, F}\right)$ is mapped onto $Q_{v}\left(a_{j}\left(R_{l-1, G}\right)\right)$. Thus $\log f^{l}$ is a bijective mapping from $F$ onto $Q_{v}\left(a_{j}\left(R_{l-1, G}\right)\right)$. Denote the inverse function by $\psi$. We collect all domains $W_{k, F} \subset$ $Q_{v}\left(a_{j}\left(R_{l-1, G}\right)\right)$ which are mapped by the exponential function onto $V\left(a_{k}\left(R_{l, F}\right)\right) \subset$ $A\left(R_{l, F}\right)$ bijectively. Then

$$
\mathcal{A}_{l+1}=\bigcup_{F \in \mathcal{A}_{l}}\left\{\psi_{F}\left(W_{k, F}\right)\right\}
$$

Thus we have finished the construction of the sets $\mathcal{A}_{l}$. To calculate the Hausdorff measure, both invariants $\Delta_{k}$ and $d_{k}$ mentioned above are needed. 
Using (4.4) and Lemma 4.5, we deduce

$$
\operatorname{area}\left(\bigcup_{k=1}^{m\left(R_{l, F}\right)} W_{k, F}\right)=m\left(R_{l, F}\right) \int_{V_{k}\left(R_{l, F}\right)} \frac{1}{|z|^{2}} d x d y \geq \frac{\eta \tau}{8} .
$$

Then (see [5, p. 549] for more details)

$$
\begin{gathered}
\operatorname{dens}\left(A_{l+1}, F\right)=\operatorname{dens}\left(\bigcup_{k=1}^{m\left(R_{l, F}\right)} \psi_{F}\left(W_{k}\left(R_{l, F}\right), \psi_{F}\left(Q_{v}\left(a_{j}\left(R_{l, G}\right)\right)\right)\right)\right. \\
\geq \frac{1}{\left(C^{\prime}\right)^{2}} \operatorname{dens}\left(\bigcup_{k=1}^{m\left(R_{l, F}\right)} W_{k}\left(R_{l, F}\right), Q_{v}\left(a_{j}\left(R_{l, G}\right)\right)\right) \geq \frac{\eta \tau}{32\left(C^{\prime}\right)^{2} \pi \log 2}=\Delta,
\end{gathered}
$$

where $C^{\prime}$ is the constant as in Lemma 4.2 .

For calculating $d_{k}$, it will be more convenient to choose any sequence of nested sets $\left\{F_{k}\right\}_{k=0}^{\infty}$ which satisfies $F_{k} \in \mathcal{A}_{k}$ and $F_{k+1} \subset F_{k}$ for every $k$. Without loss of generality, let $F_{l-1}=G$ and $F_{l}=F$, where $F, G$ are as above. In what follows, we use the abbreviated notation $R_{k}=R_{k, F_{k}}, a_{j}=a_{j}\left(R_{l-1, G}\right)$ and $V_{j}\left(R_{l-1}\right)=V\left(a_{j}\left(R_{l-1, G}\right)\right)$.

Recall the formula (4.6). Let $\phi$ be the branch of the inverse of $f^{l-1}$ which maps $f^{l-1}(F)$ to $F$. Then $\phi$ is a univalent map in the domain $D\left(a_{j}, t\left(R_{l-1}\right)\right)$ and maps its subset $V_{j}\left(R_{l-1}\right)$ onto $F$. Furthermore, $\phi$ can extend to a univalent map in $D\left(a_{j}, 2 t\left(R_{l-1}\right)\right)$ by Lemma 4.4. Koebe's distortion theorem implies that if $z \in$ $D\left(a_{j}, t\left(R_{l-1}\right)\right)$, then $\left|\phi^{\prime}(z)\right| \leq 12\left|\phi^{\prime}\left(a_{j}\right)\right|$. So

$$
\operatorname{diam}(F) \leq 12\left|\phi^{\prime}\left(a_{j}\right)\right| \operatorname{diam}\left(f^{l-1}(F)\right) \leq 24\left|\phi^{\prime}\left(a_{j}\right)\right| t\left(R_{l-1}\right) .
$$

It follows from (4.5) that

$$
\left|f^{\prime}\left(f^{k}(z)\right)\right| \geq q \frac{\left|f^{k+1}(z)\right|}{t\left(R_{k}\right)} \geq q \frac{R_{k+1}}{t\left(R_{k}\right)}=\tau_{1} \frac{R_{k+1}}{R_{k}} \log M\left(R_{k}, f\right),
$$

where $\tau_{1}=\frac{q L}{\lambda}$. Since $\left|\phi^{\prime}\left(a_{j}\right)\right|=\frac{1}{\left|\left(f^{l-1}\right)^{\prime}\left(\phi\left(a_{j}\right)\right)\right|}$ and $\left(f^{l-1}\right)^{\prime}(z)=\prod_{k=0}^{l-2} f^{\prime}\left(f^{k}(z)\right)$, we conclude that

$$
\left|\phi^{\prime}\left(a_{j}\right)\right| \leq \frac{R_{0}}{R_{l-1}} \prod_{k=0}^{l-2} \frac{1}{\tau_{1} \log M\left(R_{k}, f\right)}
$$

and thus

$$
\begin{aligned}
\operatorname{diam}(F) & \leq 24\left|\phi^{\prime}\left(a_{j}\right)\right| t\left(R_{l-1}\right) \\
& \leq 24 \frac{R_{0} t\left(R_{l-1}\right)}{R_{l-1}} \prod_{k=0}^{l-2} \frac{1}{\tau_{1} \log M\left(R_{k}, f\right)}=\prod_{k=0}^{l-1} \frac{\tau_{2}}{\tau_{1} \log M\left(R_{k}, f\right)},
\end{aligned}
$$

where $\tau_{2}$ is a constant. From the condition (2.2), we have

$$
R_{k} \geq \exp \left(R_{k-1}^{\delta}\right)=E^{k}\left(R_{0}\right)
$$

where $E^{k}\left(R_{0}\right)$ is the $k$-th iteration of $\exp \left(z^{\delta}\right)$ in point $R_{0}$.

For $\lambda_{0} \in(0,1 / e)$, we denote $E_{\lambda_{0}}^{k}(z)$ by the $k$-th iteration of exponential $\lambda_{0} \exp (z)$. Fix $r_{0}$, then for every $\lambda_{0} \in \mathbf{C}$ and $l \in \mathbf{N}$, there exists $r_{1}$ such that $E^{l}\left(r_{1}\right) \geq E_{\lambda_{0}}^{l}\left(r_{0}\right)$. 
Thus for $l \geq 2$ and sufficiently large $R_{0}$, we have

$$
\begin{aligned}
\operatorname{diam}\left(F_{l}\right) & \leq \prod_{k=0}^{l-1} \frac{\tau_{2}}{\tau_{1} \log M\left(R_{k}, f\right)} \leq \prod_{k=0}^{l-1} \frac{1}{\log R_{k}} \\
& \leq \frac{1}{\log E^{l-1}\left(R_{0}\right)} \leq \frac{1}{\log E_{\lambda_{0}}^{l-1}\left(r_{0}\right)} \leq \frac{1}{E_{\lambda_{0}}^{l-1}\left(r_{0}^{\prime}\right)}=d_{l}
\end{aligned}
$$

where $r_{0}^{\prime}<r_{0}$. Since we can take $r_{0}^{\prime}, r_{0} \in U$, where $U$ be as in (1.1). It follows from (1.1), (4.7) and (4.8) that

$$
\Phi\left(\frac{1}{d_{l}}\right)^{\kappa} \prod_{j=1}^{l} \Delta_{j}=\Phi\left(E_{\lambda_{0}}^{l-2}\left(r_{0}^{\prime}\right)\right)^{\kappa} \Delta^{l}=\left(\beta_{\lambda_{0}}^{l-2} \Phi\left(r_{0}^{\prime}\right)\right)^{\kappa} \Delta^{l}=\left(\beta_{\lambda_{0}}^{\kappa} \Delta\right)^{l-2} \Phi\left(r_{0}^{\prime}\right)^{\kappa} \Delta^{2},
$$

which tends to $\infty$ as $l$ tends to $\infty$ when $\beta_{\lambda_{0}}^{\kappa} \Delta>1$. Thus Lemma 4.3 implies that for $\kappa>\frac{\log (1 / \Delta)}{\log \beta_{\lambda_{0}}}$

$$
H^{h}(A)=\infty, \quad \text { where } h(t)=t^{2} \Phi\left(\frac{1}{t}\right)^{\kappa}
$$

Moreover, from Lemma 4.6 we get $A=\bigcap_{l=1}^{\infty} A_{l} \subset J(f)$. Hence $A \subset I(f) \cap J(f)$ since $A \subset I(f)$ by (2.2). Thus

$$
H^{h}(I(f) \cap J(f))=\infty .
$$

This completes the proof of Theorem 2.1.

4.3. Proof of Corollary 2.2. Obviously, $\log ^{m}\left(\frac{1}{d_{l}}\right) \prod_{j=1}^{l} \Delta_{j}$ tends to infinity as $l \rightarrow \infty$. Using Lemma 4.3 to it, we can complete the proof of Corollary 2.2.

Acknowledgements. The author would like to thank Walter Bergweiler for suggesting that the assertion in Theorem 2.1 should be true, and for his help and encouragement. The author is also grateful to Janne Heittokangas for helpful comments.

\section{References}

[1] Aspenberg, M., and W. Bergweiler: Entire functions with Julia sets of positive measure. - Math. Ann. 352, 2012, 27-54.

[2] Barański, K.: Hausdorff dimension of hairs and ends for entire maps of finite order. - Math. Proc. Cambridge Philos. Soc. 145, 2008, 719-737.

[3] Beardon, A. F.: Iteration of rational functions. - Spinger, Berlin, 1991.

[4] Bergweiler, W.: Iteration of meromorphic functions. - Bull. Amer. Math. Soc. (N.S.) 29, 1993, 151-188.

[5] Bergweiler, W., and B. Karpińska: On the Hausdorff dimension of the Julia set of a regularly growing entire function. - Math. Proc. Cambridge Philos. Soc. 148, 2010, 531-551.

[6] Bergweiler, W., B. Karpińska, and G. M. Stallard: The growth rate of an entire function and the Hausdorff dimension of its Julia set. - J. London Math. Soc. 80:2, 2009, $680-698$.

[7] Bergweiler, W., and J. Peter: Escape rate and Hausdorff measure for entire functions. Math. Z. 274, 2013, 551-572.

[8] BoAs, R. P., JR: Entire functions. - Academic Press, New York, 1954.

[9] Clunie, J.: On integral functions having prescribed asymptotic growth. - Canad. J. Math. 17, $1965,396-404$.

[10] Eremenko, A. E.: On the iteration of entire functions. - In: Dynamical Systems and Ergodic Theory, Banach Center Publications 23, Polish Scientific Publishers, Warsaw, 1989, 339-345. 
[11] Eremenko, A.E., and M. Yu. Lyubich: Dynamical properties of some classes of entire functions. - Ann. Inst. Fourier (Grenoble) 42, 1992, 989-1020.

[12] Goldberg, A.A., and I. V. OstrovskiI: Value distribution of meromorphic functions. Transl. Math. Monogr. 236, Amer. Math. Soc., 2008.

[13] Hayman, W. K.: Meromorphic functions. - Clarendon Press, Oxford, U.K., 1964.

[14] Kotus, J., and M. URBAŃski: Fractal measures and ergodic theory of transcendental meromorphic functions. - In: Transcendental Dynamics and Complex Analysis (edited by P. J. Rippon and G. M. Stallard), London Math. Soc. Lecture Note Ser. 348, Cambridge Univ. Press, Cambridge, 2008, 251-316.

[15] Levin, B. JA.: Distribution of zeros of entire functions. Revised edition. - Transl. Math. Monogr. 5, Amer. Math. Soc., Providence, R.I., 1980.

[16] Macintyre, A. J., and W. H. J. Fuchs: Inequalities for the logarithmic derivatives of a polynomial. - J. London Math. Soc. 15, 1940, 162-168.

[17] McMullen, C.: Area and Hausdorff dimension of Julia sets of entire functions. - Trans. Amer. Math. Soc. 300, 1987, 329-342.

[18] Milnor, J.: Dynamics in one complex variable. - Friedr. Vieweg \& Sohn, Braunschweig, 1999.

[19] Peter, J.: Hausdorff measure of escaping and Julia sets for bounded-type functions of finite order. - Ergodic Theory Dynam. Systems 33, 2013, 284-302.

[20] Peter, J.: Hausdorff measure of Julia sets in the exponential family. - J. London Math. Soc. 82:1, 2010, 229-255.

[21] Rempe, L., and G. M. Stallard: Hausdorff dimensions of escaping sets of transcendental entire functions. - Proc. Amer. Math. Soc. 138, 2010, 1657-1665.

[22] Schubert, H.: Über die Hausdorff-Dimension der Juliamenge von Funktionen endlicher Ordnung. - Dissertation, University of Kiel, 2007.

[23] Stallard, G. M.: Dimensions of Julia sets of transcendental meromorphic functions. - In: Transcendental Dynamics and Complex Analysis (edited by P. J. Rippon and G. M. Stallard), London Math. Soc. Lecture Note Ser. 348, Cambridge Univ. Press, Cambridge, 2008, 425-446.

[24] Stallard, G. M.: The Hausdorff dimension of Julia sets of entire functions II. - Math. Proc. Cambridge Philos. Soc. 119, 1996, 513-536.

[25] Taniguchi, M.: Size of the Julia set of structurally finite transcendental entire function. Math. Proc. Cambridge Philos. Soc. 135, 2003, 181-192.

[26] Zheng, J. H.: Dynamics of Transcendental Meromorphic Functions. - Tsinghua Univ. Press, Beijing, 2006 (in Chinese).

[27] ZHENG, J. H.: On multiply-connected Fatou components in iteration of meromorphic functions. - J. Math. Anal. Appl. 313, 2006, 24-37.

Received 18 April 2016• Accepted 5 August 2016 